This is the author's version of an article that has been published in the ICIT 2018 proceedings.

Changes were made to this version by the publisher prior to publication.

The final version of record is available at https://dx.doi.org/10.1109/ICIT.2018.8352183

\title{
Modeling and Experimental Validation of the Influence of Robot Temperature on Its Energy Consumption
}

\author{
Kai Eggers, Elias Knöchelmann, Svenja Tappe, Tobias Ortmaier \\ Gottfried Wilhelm Leibniz Universität Hannover \\ Institute of Mechatronic Systems \\ Hanover, Germany \\ Email: kai.eggers@imes.uni-hannover.de
}

\begin{abstract}
This paper presents an approach to significantly improve modeling accuracy for the power and energy demands of industrial robots. This is achieved by taking the temperature dependency of the joint's viscuous friction parameters into account. While the connection is commonly known, it is usually neglected in state-of-the-art energy consumption models for industrial robots. This paper shows that a consideration of temperature-dependent friction provides significant improvement of energy modeling accuracy. The approach is validated on a test rig with a KUKA KR 16 robotic manipulator. Measurements show that the grid energy consumption modeling error can be reduced from up to $45 \%$ to approx. $5 \%$ over the whole spectrum of operating temperatures.
\end{abstract}

Index Terms-Industrial Robotics, Energy Efficiency, Robot Temperature, Friction Modeling

\section{INTRODUCTION}

Sales of industrial robots reached an all time high of 248,000 units sold in 2015 worldwide, increasing by about $12 \%$ compared to the previous year [1]. Ongoing growth is predicted for the future. However, a rising degree of automation also leads to an increase of overall energy consumption. Recent research addresses this topic by developing methods for increasing energy efficiency of components, machines, and/or the whole manufacturing process.

Especially the software-based approaches that try to calculate e.g. the most efficient scheduling for a robot cell [2], [3] or the energy-optimal trajectory for single robots [4], rely on modeling the system's energy consumption in order to find favorable operating points. Therefore, the energy model's accuracy directly affects the results of said calculations. For complex machines like industrial robots, the consumption depends on several factors, e.g. the regarded motion including velocities and motor torques. Further, the energy consumption is significantly influenced by robot temperature, that varies from approx. 20 to $80^{\circ} \mathrm{C}$ depending on its degree of utilization. The presented paper aims on improving the modeling accuracy for the energy consumption of industrial robots by taking this dependency into account. Validation measurements show that, using the presented modeling enhancements, a high

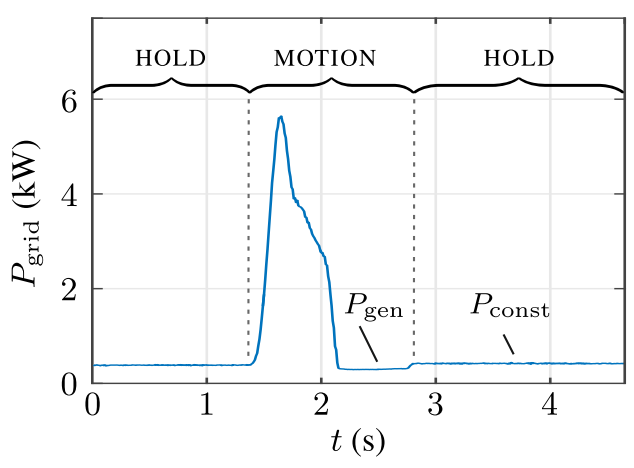

Fig. 1. Typical power consumption measurement for an industrial robot in different operating phases

modeling accuracy can be achieved over the whole operating temperature spectrum.

This article is structured as follows: The basic approach for modeling power and energy demands of industrial robots is shown in section II. The temperature-dependent identification of friction parameters is explained in section III. Measurement and validation results are presented in section IV. The paper closes with a conclusion in section $\mathrm{V}$.

\section{POWER AND ENERGY MODEL FOR INDUSTRIAL ROBOTS}

First, the state-of-the-art modeling approach is introduced. The approaches provide high accuracy and are able to correctly depict power flows of industrial robots. A typical grid power demand can be seen in Fig. 1. The measurement was performed at a KUKA KR 16 industrial robot, the test rig is described later on in section II-C. The HOLD phase shows the power demand in standstill but with active control. The motion-dependent power consumption is marked as MOTION phase.

The general modeling approach based on a substitue circuit diagram is explained in section II-A, along with the identification of the required cabinet parameters. The modeling of the 
This is the author's version of an article that has been published in the ICIT 2018 proceedings.

Changes were made to this version by the publisher prior to publication.

The final version of record is available at https://dx.doi.org/10.1109/ICIT.2018.8352183

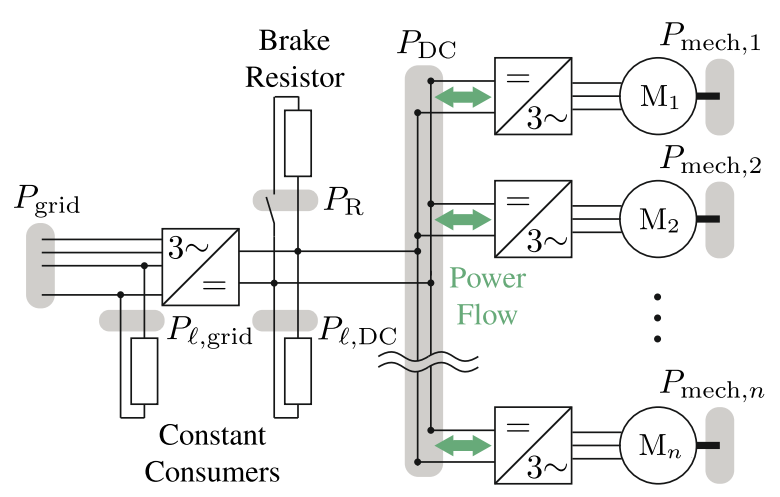

Fig. 2. Electrical substitute circuit diagram for an industrial robot

motion-dependent power consumption is formulated in section II-B. Validation measurements are presented in section II-C.

\section{A. Substitute Circuit Diagram}

The power consumption of industrial robots can be explained by consideration of a simplified electrical substitute circuit diagram (Fig. 2). Starting on the right side of the graphic, the mechanical power demand $P_{\text {mech }, i}$ is predefined by the motion and process planning. A detailed description of the modeling approach for this particular term can be found in section II-B. All axes usually share a supply module, the axes are electrically coupled via the DC bus. This enables power exchange between them. The sum of mechanical powers is represented by an auxiliary variable $P_{\mathrm{DC}}$ that characterizes this power flow. Since most state-of-the-art robot controls are not able to recuperate, excess power $P_{\mathrm{R}}$ is dissipated through a brake resistor. DC bus losses are summarized as a constant loss power $P_{\ell, \mathrm{DC}}$, while $P_{\ell \text {, grid }}$ contains the constant losses of all peripheral components (e.g. controller, cooling fans, IO modules, sensors, etc.) as well as the holding brakes that require active lifting.

In the HOLD phase, the power demand is induced by the constant losses on the grid side $P_{\ell \text {,grid }}$ and on the DC side $P_{\ell, \mathrm{DC}}$ :

$$
P_{\text {const }}=P_{\ell, \text { grid }}+P_{\ell, \mathrm{DC}} .
$$

The power consumption for the static holding is not considered separately. It is instead included in the constant DC losses $P_{\ell, \mathrm{DC}}$. The dependency on the robot configuration is neglected: for the KUKA KR 16, measurements have shown a maximum deviation of $P_{\text {hold }}$ of approx. $10 \%$ for the most extreme poses (see section II-C for the test bed description). Larger robots usually feature a counterbalancing weight system which further diminishes the influence. However, with regard to the KR 16's peak power of approx. $8 \mathrm{~kW}$ (see Fig. 4), the deviation comes down to less than $0.01 \%$.

In order to provide the desired accuracy, industrial robots usually feature backlash free gears with high friction. Hence, the mechanical losses usually clearly exceed the electrical ones. Therefore, the proposed simplifications only have a minor impact on the grid power consumption (see section II-C).

\section{B. Motion-dependent Power Consumption}

This section focuses on the calculation of the power consumption in dependence of the executed motion. Assuming that a trajectory of an industrial robot is predefined, the calculation of the grid power demand starts with determining the motor torques $\boldsymbol{\tau}(t)$. In general, the model for inverse dynamics is given by

$$
\begin{aligned}
\boldsymbol{\tau}(t)= & \operatorname{diag}\left(\frac{1}{u_{\mathrm{G}, 1}}, \ldots, \frac{1}{u_{\mathrm{G}, n}}\right)(\boldsymbol{M}(\boldsymbol{q}) \ddot{\boldsymbol{q}}+\boldsymbol{c}(\boldsymbol{q}, \dot{\boldsymbol{q}})+\boldsymbol{g}(\boldsymbol{q})) \\
& +\boldsymbol{h}(\boldsymbol{q}, \dot{\boldsymbol{q}})
\end{aligned}
$$

where $\boldsymbol{q}, \dot{\boldsymbol{q}}, \ddot{\boldsymbol{q}}$ are time-dependent joint angles, velocities, and accelerations provided by the motion planning algorithm. The term $u_{\mathrm{G}, i}$ represents the gear factor for joint $i$ while the vector $\tau$ contains the respective motor torques $\tau_{i}$. $M$ contains moments of inertia, $c$ Coriolis effects, and $\boldsymbol{g}$ gravitational effects. $\boldsymbol{h}$ summarizes non-linear effects which in our regarded case is the aforementioned friction. Several comprehensive friction models can be found in literature, e.g. [5], [6]. However, many applications feature a more simplified model that yields satisfying results. In [7], a commonly used friction model including Coulomb friction and viscous damping (coefficients $f_{\mathrm{c}, i}$ and $f_{\mathrm{v}, i}$, respectively) is presented. It is applied for this model, expressing friction torque $\tau_{\mathrm{f}, i}$ for joint $i$ as

$$
\tau_{\mathrm{f}, i}(t)=h_{i}(t)=f_{\mathrm{c}, i} \operatorname{sign}\left(\omega_{i}(t)\right)+f_{\mathrm{v}, i} \omega_{i}(t),
$$

where $\omega_{i}$ is the angular motor velocity of motor $i$ which can be determined as

$$
\omega_{i}(t)=u_{\mathrm{G}, i} \dot{q}_{i}(t) .
$$

Most robotic manufacturers utilize a model of the inverse dynamics within the robot control system for implementation of feed forward control. Thus, it can be assumed that system friction parameters are known. If not, they can be obtained using established identification methods [8] (see also section III). Equations (2) and (4) are used to obtain the mechanical power $P_{\text {mech }, i}(t)$ for each motor $i$ :

$$
P_{\text {mech }, i}(t)=\tau_{i}(t) \omega_{i}(t) .
$$

The total DC bus power $P_{\mathrm{DC}}$ is obtained by summing up the mechanical power of the $n$ individual motors:

$$
P_{\mathrm{DC}}(t)=\sum_{i=1}^{n} P_{\text {mech }, i}(t) .
$$

For state-of-the-art industrial robots, the DC bus features a capacitor that is usually dimensioned to smoothen rectified voltage, not to buffer excess energy in generator operation phases. Therefore, the capacity is neglected. However, it can be implemented according to [4] if desired. Further, rectifiers in industrial robot cabinets are usually not able to recuperate. Hence, negative values for $P_{\mathrm{DC}}$ need to be partly corrected. The excess power in generator operating phases can cover constant losses within the DC bus, but grid side losses will remain. The remaining power consumption is marked as $P_{\text {gen }}$ in Fig. 1. This is considered as follows: 
This is the author's version of an article that has been published in the ICIT 2018 proceedings.

Changes were made to this version by the publisher prior to publication.

The final version of record is available at https://dx.doi.org/10.1109/ICIT.2018.8352183

$$
\begin{aligned}
P_{\mathrm{DC}}(t)+P_{\ell, \mathrm{DC}} \geq 0 & : \\
P_{\text {grid }}(t) & =P_{\mathrm{DC}}(t)+P_{\ell, \mathrm{DC}}+P_{\ell, \text { grid }}, \\
P_{\mathrm{R}}(t) & =0 \\
P_{\mathrm{DC}}(t)+P_{\ell, \mathrm{DC}}<0 & : \\
P_{\text {grid }}(t) & =P_{\text {gen }}=P_{\ell, \text { grid }}, \\
P_{\mathrm{R}}(t) & =\left|P_{\mathrm{DC}}(t)+P_{\ell, \mathrm{DC}}\right|
\end{aligned}
$$

where $P_{\mathrm{R}}(t)$ is the power dissipated via the brake resistor. The time integral of the grid power demand over trajectory time (from $t_{\text {start }}$ to $t_{\text {end }}$ )

$$
E_{\text {grid }}=\int_{t_{\text {start }}}^{t_{\text {end }}} P_{\text {grid }}(t) \mathrm{d} t
$$

equals the grid energy demand $E_{\text {grid }}$ of the system for the respective motion.

\section{Validation of Temperature Dependency}

The model is applied to a KUKA KR 16 with a maximum payload of $16 \mathrm{~kg}$. The test setup for the validation is shown in Fig. 3. The grid power is measured using a Yokogawa WT 3000 precision power analyzer. The robot is equipped with a test weight of $15 \mathrm{~kg}$.

If the robot has a predefined thermal state, the presented modeling approach grants a high model accuracy, as shown in Fig. 4. However, this is only viable for laboratory conditions, not for industrial applications, where the robot temperature depends on its degree of utilization. Figure 5 demonstrates the impact of the robot (i. e. motor) temperature on mechanical power demand. A higher temperature leads to a decrease of gearbox oil viscosity and, subsequently, to a reduction of mechanical losses. The values are based on the traced values at a KR 16 using its internal motor sensors for currents (torques), velocities, and temperatures. While the motor temperature $\vartheta_{i}$ does not necessarily equal the gear temperature, it sufficiently displays the robot's relative thermal state using built-in sensors. The measured grid energy consumption for the same motion at different temperatures is shown in section IV.

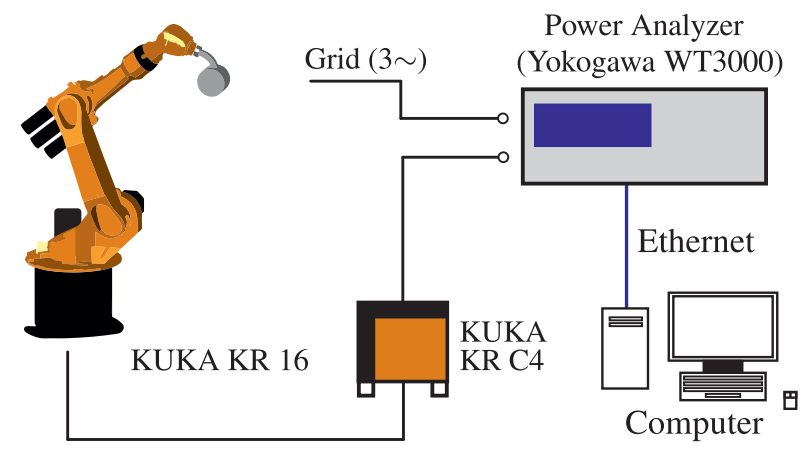

Fig. 3. Test setting for power and energy measurements
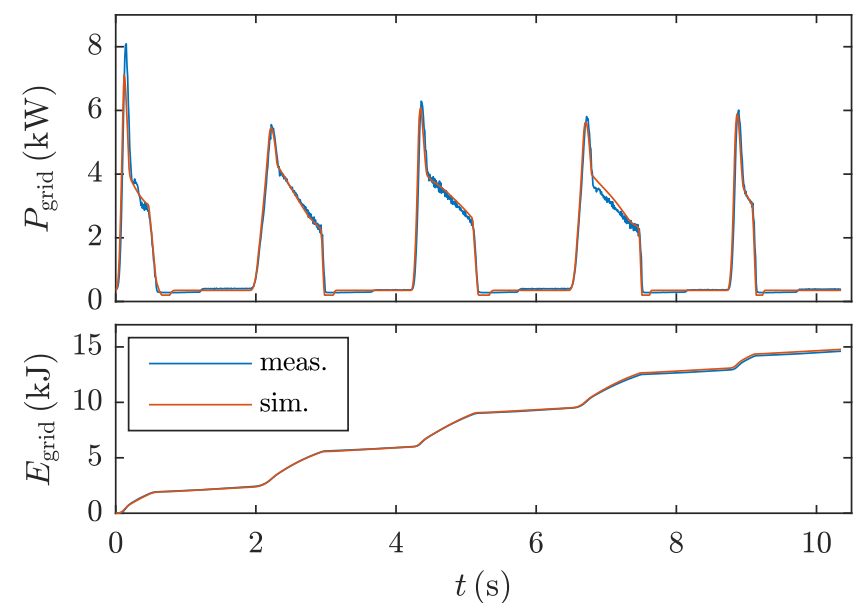

Fig. 4. Measured and simulated power demand (top) and energy demand (bottom) for the KUKA KR 16 with $\vartheta=60^{\circ} \mathrm{C}$

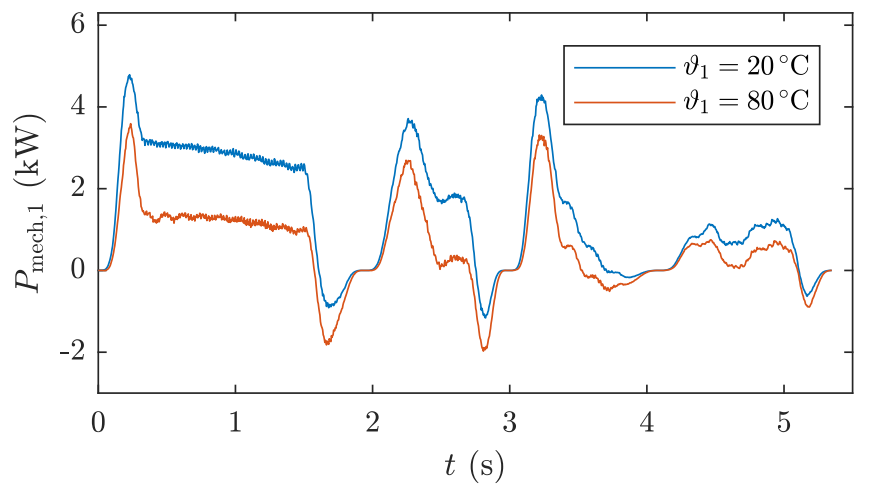

Fig. 5. Mechanical power comparison for axis $i=1$ at different motor temperatures based on measured (traced) values $\widetilde{\tau}_{1}$ and $\widetilde{\omega}_{1}$

It becomes obvious that a consideration of the temperature within the model is inevitable, but previous works often neglect this. While [9], [10] consider the robot temperature, only temperature dependencies of motor resistors are taken into account. However, it will be demonstrated that the dependency of power consumption on temperature can be adequately depicted by adjusting the friction parameters, as presented in sections III and IV.

\section{IDENTIFICATION OF TEMPERATURE-DEPENDENT FRICTION PARAMETERS}

This section describes the approach to correctly depict the behaviour presented in Fig. 5 in the energy model. For this, the previously introduced friction model (3) will be expanded. In [11], it is stated that $f_{\mathrm{C}, i}$ is not affected by temperature changes, but $f_{\mathrm{v}, i}$ holds a logarithmic dependency. Therefore, only $f_{\mathrm{v}, i}$ will be modeled as temperature-dependent:

Copyright (c) 2019 IEEE. Personal use of this material is permitted. For any other purposes, permission must be obtained from the IEEE by emailing pubs-permissions@ieee.org. 
This is the author's version of an article that has been published in the ICIT 2018 proceedings.

Changes were made to this version by the publisher prior to publication.

The final version of record is available at https://dx.doi.org/10.1109/ICIT.2018.8352183

$$
\tau_{\mathrm{f}, i}(t)=f_{\mathrm{c}, i} \operatorname{sign}\left(\omega_{i}(t)\right)+f_{\mathrm{v}, i}\left(\vartheta_{i}\right) \omega_{i}(t) .
$$

Now, $f_{\mathrm{v}, i}$ is identified for each axis on varying temperatures, utilizing the test rig described in II-C. For identification, the robot executes a warm-up program until the specified motor temperatures are reached. Actual motor torque values $\widetilde{\tau}_{i}$ are traced as provided by the robot controller. Values for $f_{\mathrm{v}, i}$ are obtained by solving the linear least-square problem for each axis $i$ :

$$
\begin{aligned}
f_{\mathrm{v}, i}(\vartheta) & =\min _{f_{\mathrm{v}, i}}\left\|\left(\Delta \tau_{i j}\left(\vartheta, f_{\mathrm{v}, i}\right)\right)\right\|_{2}^{2}, \text { with } \\
\Delta \tau_{i j}\left(\vartheta, f_{\mathrm{v}, i}\right) & =\tau_{i j}\left(\vartheta, f_{\mathrm{v}, i}\right)-\widetilde{\tau}_{i j}(\vartheta),
\end{aligned}
$$

where $j$ represents discrete time step and $m$ the number of time steps within the measurement. Friction identification by solving a least-square problem is a common approach. More detailed information can be found in [12]. The identified temperature-dependent viscuous friction coefficients can be used to fit an exponential function or saved directly.

\section{RESULTS}

The method introduced in section III is applied to a KUKA KR 16 industrial robot. The robot executes a set of 20 PTP motions and values for $\widetilde{\tau}_{i}$ are traced for all axes. The viscuous damping parameters are identified according to (9).

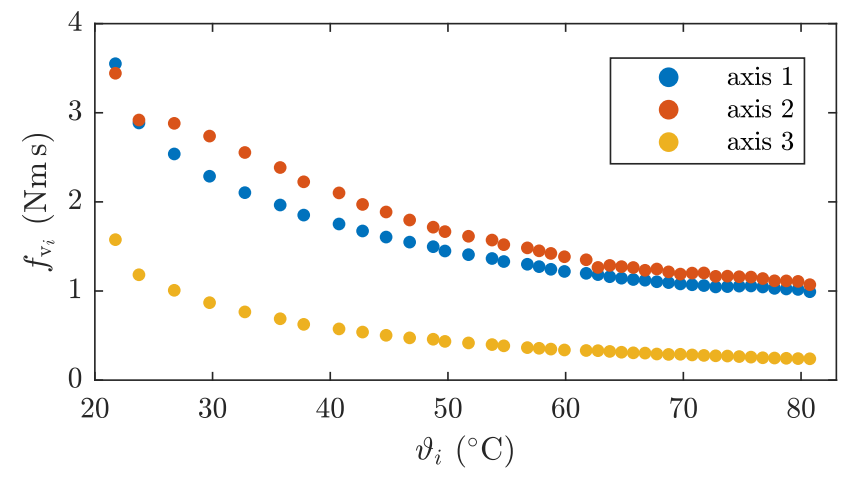

(a) $f_{\mathrm{v}, i}$ for main axes $i=1 . .3$

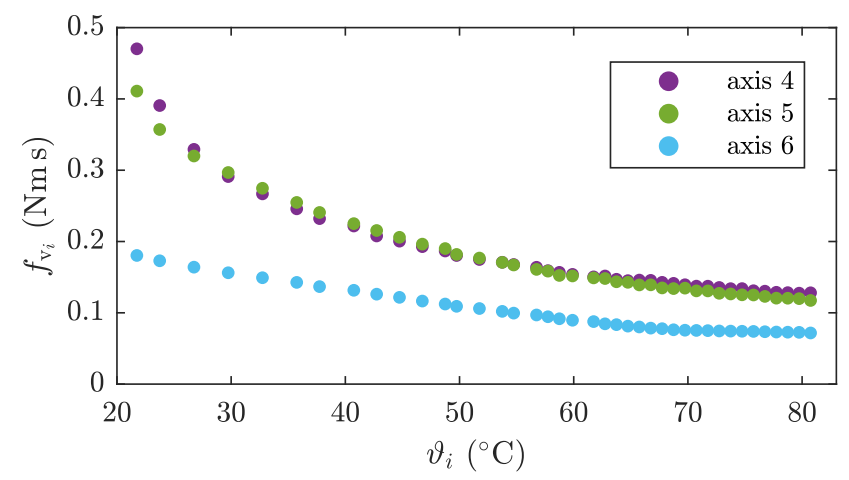

(b) $f_{\mathrm{v}, i}$ for hand axes $i=4 . .6$

Fig. 6. Identified viscuous damping $f_{\mathrm{v}, i}$ over motor temperature $\vartheta_{i}$ for all axes $i=1 . .6$
Then, robot temperature is raised by performing a designated warm-up motion for one minute, followed by a new measurement with the same set of PTP motions, and so forth. The identified damping parameters are shown in Fig. 6. Values at very low temperatures feature a slight systematic error because robot temperature rises up to two degrees during measurement. The modeled energy consumption over robot temperature is displayed in Fig. 7a, along with the measured grid energy demand (measured with the test setting shown in Fig. 3). The corresponding model deviation is shown in Fig. 7b. Since the original approach utilizes friction parameters of a fixed operating point, the modeled energy consumption is not affected by changes of robot temperature. In contrast, it can be seen that the presented approach enables high modeling accuracy across the whole operating temperature spectrum.

The remaining model deviation of approx. $5 \%$ mainly results from deviations of torque set and actual values as provided by the robot controller. The torque error emerges from parameter dispersion for all components as well as several model simplifications, such as the negligence of structural and drive oscillations, influence of drive control, and motor demagnetization effects.

\section{CONCLUSION}

This paper presented an approach to increase modeling accuracy for the energy consumption of industrial robots.

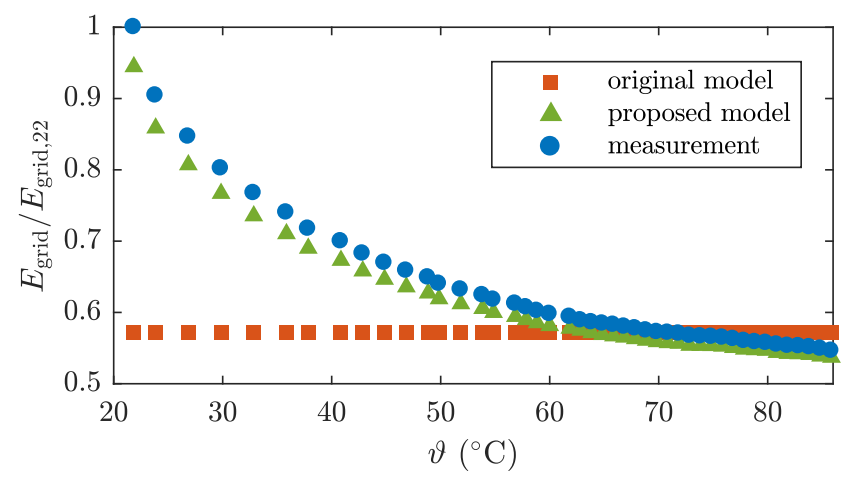

(a) Dependency of $E_{\text {grid }}$ on temperature, normalized to $E_{\text {grid }, 22}$ at $22{ }^{\circ} \mathrm{C}$

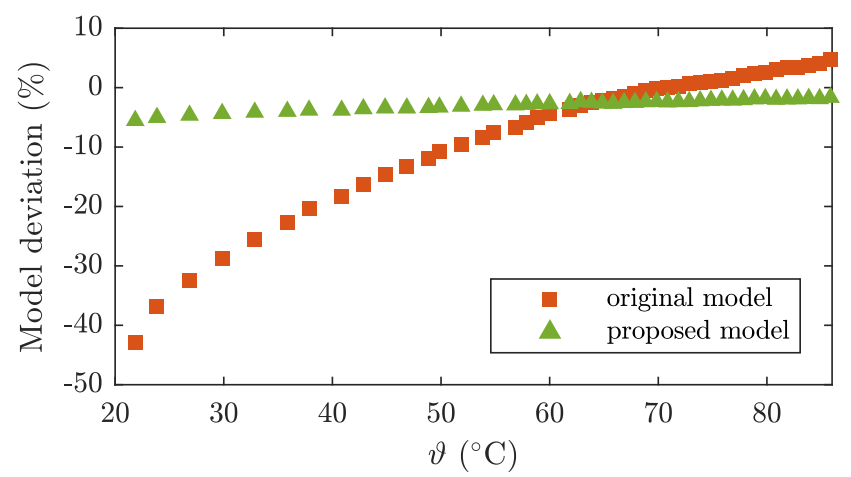

(b) Model deviation in \% for original (red) and proposed model (green)

Fig. 7. Comparison of measured and modeled grid energy demand at varying robot temperatures

Copyright (c) 2019 IEEE. Personal use of this material is permitted. For any other purposes, permission must be obtained from the IEEE by emailing pubs-permissions@iee.org. 
This is the author's version of an article that has been published in the ICIT 2018 proceedings.

Changes were made to this version by the publisher prior to publication.

The final version of record is available at https://dx.doi.org/10.1109/ICIT.2018.8352183

The improvement is achieved by taking the robot temperature into account. In detail, the viscuous damping parameters are modified based on the motor temperature of each axis. Validation measurements have shown that the approach significantly increases modeling accuracy across the whole operating temperature spectrum. The enhanced model can be applied to existing efficiency optimization approaches, where a more accurate model yields better (i.e. more energy-efficient) results.

\section{ACKNOWLEDGMENT}

The presented research is funded by German Research Foundation (Deutsche Forschungsgemeinschaft/DFG).

\section{REFERENCES}

[1] World Record: 248.000 Industrial Robots Revolutionising the Global Economy, IFR press release, International Federation of Robotics Std., 06 2016. [Online]. Available: http://www.ifr.org/news/ifr-pressrelease/world-record-816/

[2] D. Meike, M. Pellicciari, G. Berselli, A. Vergnano, and L. Ribickis, "Increasing the energy efficiency of multi-robot production lines in the automotive industry," in Proc. of the IEEE International Conference on Automation Science and Engineering, 2012, pp. 700-705.

[3] J. Greenyer and D. Gritzner, "An approach for synthesizing energyefficient controllers for production systems from scenario-based specifications." in D\&P@MoDELS, 2016, pp. 87-93.

[4] C. Hansen, J. Öltjen, D. Meike, and T. Ortmaier, "Enhanced Approach for Energy-Efficient Trajectory Generation of Industrial Robots," in Proc. of the IEEE International Conference on Automation Science and Engineering, 2012, pp. 1-7.
[5] H. Olsson, K. strm, C. C. de Wit, M. Gfvert, and P. Lischinsky, "Friction models and friction compensation," European Journal of Control, vol. 4, no. 3, pp. 176-195, 1998. [Online]. Available: http://www.sciencedirect.com/science/article/pii/S094735809870113X

[6] N. A. Bompos, P. K. Artemiadis, A. S. Oikonomopoulos, and K. J. Kyriakopoulos, "Modeling, full identification and control of the mitsubishi pa-10 robot arm," in 2007 IEEE/ASME international conference on advanced intelligent mechatronics, Sept 2007, pp. 1-6.

[7] P. Hamon, M. Gautier, and P. Garrec, "Dynamic Identification of Robots With a Dry Friction Model Depending on Load and Velocity," in Proc. of the IEEE/RSJ International Conference on Intelligent Robots and Systems, 2010, pp. 6187-6193.

[8] C. T. Johnson and R. D. Lorenz, "Experimental identification of friction and its compensation in precise, position controlled mechanisms," IEEE Transactions on Industry Applications, vol. 28, no. 6, pp. 1392-1398, 1992.

[9] M. Brossog, M. Bornschlegl, J. Franke et al., "Reducing the Energy Consumption of Industrial Robots in Manufacturing Systems," The International Journal of Advanced Manufacturing Technology, vol. 78, no. 5-8, pp. 1315-1328, 2015.

[10] D. Meike, M. Pellicciari, and G. Berselli, "Energy Efficient Use of Multirobot Production Lines in the Automotive Industry: Detailed System Modeling and Optimization," IEEE Transactions on Automation Science and Engineering, vol. 11, no. 3, pp. 798-809, 2014.

[11] H. Wittel, D. Muhs, D. Jannasch, and J. Voiek, Roloff/Matek Maschinenelemente - Normung, Berechnung, Gestaltung - Lehrbuch und Tabellenbuch, 19th ed. Berlin Heidelberg New York: Springer-Verlag, 2009.

[12] M. Gautier, P. O. Vandanjon, and C. Presse, "Identification of inertial and drive gain parameters of robots," in Proceedings of 1994 33rd IEEE Conference on Decision and Control, vol. 4, Dec 1994, pp. 3764-3769. 